\title{
Low Complexity Joint Semiblind Detection for OFDM Systems over Time-Varying Channels
}

\author{
Y. H. Zhang, W.-S. Lu, and T. A. Gulliver \\ Department of Electrical and Computer Engineering, University of Victoria \\ P.O. Box 3055, STN CSC, Victoria, B.C., Canada V8W 3P6 \\ Email: yhzhang,wslu, agullive@ece.uvic.ca
}

\begin{abstract}
Orthogonal frequency division multiplexing (OFDM) modulation is widely used in communication systems to meet the demand for ever increasing data rates. In this paper, a low complexity joint semiblind detection algorithm for OFDM systems over time-varying channels is proposed based on the channel correlation and noise variance. The problem is relaxed to a continuous non-convex quadratic programming problem. Then an iterative method is utilized to deduce a sequence of reduced-size quadratic programming problems. These are solved by limiting the search in the 2-dimensional subspace. Furthermore, a low-bit descent search is employed to improve the system performance. Results are given which demonstrate that the proposed algorithm provides comparable performance with lower computational complexity than that of a sphere decoder.
\end{abstract}

\section{INTRODUCTION}

Orthogonal frequency division multiplexing (OFDM) modulation is widely used in communication systems to efficiently provide high data rates. The major advantage of OFDM over single-carrier transmission is its ability to deal with severe channel conditions without complex equalization. The standards employing OFDM modulation include digital video broadcasting (DVB) [1], digital audio broadcasting DAB [2], IEEE 802.11 [3] for wireless local area networks, and IEEE 802.16 [4] for wireless metropolitan area networks.

In an OFDM system, the data stream is modulated on orthogonal subcarriers using phase-shift keying (PSK) or quadrature amplitude modulation (QAM). OFDM systems are sensitive to Doppler spread caused by user mobility, which results in loss of orthogonality among subcarriers. This leads to intercarrier interference (ICI) which degrades system performance.

In the design of most ICI reduction algorithms for OFDM systems [5]-[6], perfect channel information is assumed at the receiver. Many channel estimation methods have been proposed for OFDM systems based on pilot symbols [7] [8]. In most cases, channel estimation with pilot symbols is a robust method. However, there are disadvantages such as bandwidth loss and overhead, which can be excessive in fast fading channels. This motivates the use of joint blind or semiblind detection methods for OFDM systems. These methods exploit the statistical or deterministic properties of the channel to detect the transmitted signals without estimating the channel [9]-[11].
There have been several recent investigations on the joint channel estimation and data detection problem in communication systems. In [9], a joint maximum-likelihood (ML) channel estimation and signal detection problem for singleinput multiple-output (SIMO) systems was formulated and solved via sphere decoding. A blind joint detection problem was formulated in [10] as an integer programming problem based on a regression model. Cui and Tellambura [11] proposed three ML data detectors for OFDM systems over fast fading channels with the assumption of known channel correlation.

A sphere decoder for joint semiblind detection provides nearoptimal performance with low computational complexity compared to an exhaustive search [11]. However, the complexity is still high, so there is need for a suboptimal detector which yields a fast solution with good performance. In this paper, an iterative joint semiblind detection algorithm (IJSD) for OFDM systems over time-varying channels is proposed. The detection problem is first relaxed to a continuous non-convex quadratic programming $(\mathrm{QP})$ problem. A sequence of reduced-size $\mathrm{QP}$ problems are then deduced and solved by limiting the search in the 2-dimensional subspace spanned by its steepest-descent and Newton directions to reduce the computational complexity. Furthermore, a low-bit descent search (LBDS) is employed to improve the system performance.

\section{SySTEM MOdEL}

Here we consider the problem of joint ML channel estimation and signal detection in an $N$-subcarrier OFDM system, where the channel is time-varying within one OFDM symbol duration, and the channel information is partly known, i.e., the channel correlation and noise variance.

In an OFDM system, the transmitted signal is generated using an inverse fast Fourier transform (IFFT)

$$
x_{n}=\frac{1}{\sqrt{N}} \sum_{k=0}^{N-1} X_{k} \exp \left(\frac{j 2 \pi k n}{N}\right) \text { for } n=0, \ldots, N-1
$$

where $x_{n}$ is the time-domain signal at the $n$th sampling instant, and $X_{k}$ is the frequency-domain data symbol for the $k$ th subcarrier. Equation (1) can be written in vector form as

$$
\mathbf{x}=\mathbf{F X}
$$


where $\mathbf{x}=\left[\begin{array}{llll}x_{0} & x_{1} & \ldots & x_{N-1}\end{array}\right]^{T}$ and $\mathbf{X}=\left[\begin{array}{llll}X_{0} & X_{1} & \ldots & X_{N-1}\end{array}\right]^{T}$ represent the time-domain and frequency-domain OFDM symbols, respectively, and $(\cdot)^{T}$ denotes transpose of a vector or matrix. $\mathbf{F}$ is the IFFT matrix with elements $f_{n, k}=$ $\frac{1}{\sqrt{N}} \exp \left(\frac{j 2 \pi k n}{N}\right)$. The OFDM symbol duration is denoted by $T_{s}$, so the chip duration of each subchannel is $T_{c}=T_{s} / N$.

In this paper, we consider a doubly selective fading channel model [12]. Thus, we have a wide sense stationary uncorrelated scattering (WSSUS) channel with impulse response given by

$$
h(t ; \tau)=\sum_{l=0}^{L-1} h\left(t ; \tau_{l}\right) \delta\left(\tau-\tau_{l}\right)
$$

where $\tau_{l}$ is the $l$ th path delay with $\tau_{0}<\tau_{1}<\ldots<\tau_{L-1}$, and $h\left(t ; \tau_{l}\right)$ is a complex Gaussian process with zero mean and variance $\sigma_{l}^{2}$. In a rich scattering environment, the channel autocorrelation function is separable in terms of time and delay [12], where the time-correlation function of the channel is characterized based on Jakes' model [13] as

$$
E\left\{h_{l_{1}}(t+\Delta t) h_{l_{2}}^{*}(t)\right\}=\sigma_{l}^{2} r_{t}(\Delta t) \delta\left(l_{1}-l_{2}\right)
$$

where $r_{t}\left(\Delta_{t}\right)=J_{0}\left(2 \pi f_{d} \Delta_{t}\right)$, and $J_{0}(\cdot)$ denotes the zerothorder Bessel function of the first kind. The frequency-domain correlation of the channel is given by

$$
r_{f}(\Delta f)=\sum_{l=0}^{L-1} \sigma_{l}^{2} e^{-j 2 \pi \Delta f \tau_{l}}
$$

For simplicity, we assume $\tau_{l}=l T_{c}$.

Generally a cyclic prefix $(\mathrm{CP})$ is inserted at the beginning of each OFDM symbol before transmission. The length $N_{c p}$ of the cyclic prefix is greater than or equal to that of the channel impulse response to eliminate intersymbol interference. Consequently, the discrete signal at the receiver can be expressed as

$$
y_{n}=\sum_{l=0}^{L-1} h(n, l) x(n-l)+w_{n} \text { for } n=-N_{c p}, \ldots, N-1
$$

where $w_{n}$ is additive white Gaussian noise (AWGN) with zero mean and variance $\sigma^{2}$.

After removing the $\mathrm{CP}$ and performing a fast Fourier transform (FFT), we obtain

$$
\mathbf{Y}=\mathbf{A X}+\mathbf{W}
$$

where $\mathbf{Y}=\left[\begin{array}{lll}Y_{0} & \ldots Y_{N-1}\end{array}\right]^{T}$ is the frequency-domain received signal, $\mathbf{A}=\mathbf{F}^{H} \mathbf{H F}, \mathbf{W}=\mathbf{F}^{H} \mathbf{w}$, and $(\cdot)^{H}$ denotes conjugate transpose of a vector or matrix. $\mathbf{H}$ and $\mathbf{w}$ denote the timedomain channel matrix and AWGN noise, respectively,

If $h\left(t ; \tau_{l}\right)$ in (3) remains constant within one OFDM symbol duration, then $\mathbf{A}$ is a diagonal matrix, and no ICI will occur. Conversely, if the channel varies within one OFDM symbol, the orthogonality of the subcarriers does not hold, and the received signal contains both the transmitted signal and ICI from other subcarriers [7]. In this case, the received signal on the $k$ th subcarrier is

$$
Y_{k}=A_{k, k} X_{k}+\sum_{m=0, m \neq k}^{N-1} A_{k, m} X_{m}+W_{k}
$$

where $k=0, \ldots, N-1, A_{k, m}$ denotes the $(k, m)$ th element of $\mathbf{A}$ and $\sum_{m=0, m \neq k}^{N-1} A_{k, m} X_{m}$ represents the ICI caused by other subcarriers.

According to the central limit theorem, the received signal $\mathbf{Y}$ can be modeled as i.i.d. zero mean complex Gaussian random variables. Thus, the autocorrelation matrix of $\mathbf{Y}$ in (7) can be written as [11]

$$
\mathbf{R}_{\mathbf{Y Y}}=\sigma_{I C I}^{2}\left(\mathbf{X}_{D} \mathbf{R}_{f} \mathbf{X}_{D}^{H}+\sigma_{e n}^{2} \mathbf{I}_{N}\right)
$$

where $\mathbf{X}_{D}=\operatorname{diag}(\mathbf{X})$

$$
\begin{gathered}
{\left[\mathbf{R}_{f}\right]_{(i, j)}=r_{f}\left((i-j) / N T_{c}\right)} \\
\sigma_{I C I}^{2}=\frac{1}{N^{2}} \sum_{n_{1}=0}^{N-1} \sum_{n_{2}=0}^{N-1} r_{t}\left[\left(n_{1}-n_{2}\right) T_{c}\right]
\end{gathered}
$$

and

$$
\sigma_{e n}^{2}=\frac{\left(\left(\sum_{l=0}^{L-1} \sigma_{l}^{2}\right)\left(1-\sigma_{I C I}^{2}\right)+\sigma^{2}\right)}{\sigma_{I C I}^{2}}
$$

\section{Joint Semiblind Detection Problem FORMULATION}

The correlation within the received signal is utilized to develop the semiblind joint detector for OFDM systems in the frequency-domain. By maximizing the log-likelihood function of the received signal conditioned on the transmitted signal, joint semiblind detection can be formulated as the following minimization problem

$$
\begin{aligned}
\operatorname{minimize} & \mathbf{Y}^{H} \mathbf{R}_{Y Y}^{-1} \mathbf{Y} \\
\text { subject to: } & X_{k} \in \mathcal{M} \text { for } k=0, \ldots, N-1
\end{aligned}
$$

where $\mathcal{M}$ contains the symbols from the modulation used.

Assuming BPSK or QPSK modulation, we have $\left|X_{k}\right|=1$. Thus the minimization problem (13) is equivalent to the problem [11]

$$
\begin{aligned}
& \operatorname{minimize} \mathbf{X}^{T} \mathbf{Y}_{D}^{H}\left\{\sigma_{I C I}^{2}\left(\mathbf{R}_{f}+\sigma_{e n}^{2} \mathbf{I}_{N}\right)\right\}^{-1} \mathbf{Y}_{D} \mathbf{X}^{*} \\
& \text { subject to: } X_{k} \in \mathcal{M} \text { for } k=0, \ldots, N-1
\end{aligned}
$$

where $\mathbf{X}^{*}$ denotes the conjugate of the transmitted signal $\mathbf{X}$. Note that this semiblind detector exhibits a phase ambiguity, where $\mathbf{X} e^{j \phi}, \phi \in[0,2 \pi)$, satisfies (14). One or more pilot tones can be employed to compensate for this ambiguity.

Define $\hat{\mathbf{Q}}=\mathbf{Y}_{D}^{H}\left\{\sigma_{I C I}^{2}\left(\mathbf{R}_{f}+\sigma_{e n}^{2} \mathbf{I}_{N}\right)\right\}^{-1} \mathbf{Y}_{D}$, it can be seen that $\hat{\mathbf{Q}}$ is a positive definite Hermitian matrix. However, the computational effort required to solve the semiblind problem (14) increases exponentially with the number of variables. In [11], a sphere decoder was proposed to solve this problem and 
provide near-optimal performance. Although the computational complexity of the sphere decoder is reduced by examining lattice points inside a hypersphere [14], we seek a suboptimal detector which provides comparable results to that of the sphere decoder with lower computational complexity.

For simplicity, only the algorithm for a QPSK OFDM system is described, as the algorithm for BPSK can be obtained with a straightforward modification. As (14) is a complex-valued optimization problem, we convert it into the following realvalued optimization problem

$$
\begin{aligned}
& \operatorname{minimize}\|\mathbf{M z}\| \\
& \text { subject to: } z_{k} \in\left\{-\frac{\sqrt{2}}{2}, \frac{\sqrt{2}}{2}\right\} \text { for } k=0, \ldots, 2 N-1
\end{aligned}
$$

where $\mathbf{M}=\left[\begin{array}{cc}\operatorname{real}(\hat{\mathbf{M}}) & \operatorname{imag}(\hat{\mathbf{M}}) \\ \operatorname{imag}(\hat{\mathbf{M}}) & -\operatorname{real}(\hat{\mathbf{M}})\end{array}\right], \hat{\mathbf{Q}}=\hat{\mathbf{M}}^{H} \hat{\mathbf{M}}$, and $\mathbf{z}=\left[\begin{array}{c}\operatorname{real}(\mathbf{X}) \\ \operatorname{imag}(\mathbf{X})\end{array}\right]$.

Clearly, the above problem is equivalent to a quadratic programming problem of the form

$$
\text { minimize } \mathbf{z}^{T} \mathbf{Q z}
$$

subject to: $z_{k} \in\{-1,1\}$ for $k=0, \ldots, 2 N-1$

where $\mathbf{Q}=\mathbf{M}^{T} \mathbf{M}$.

Problem (16) is a combinatorial optimization problem with exponential computational complexity. This type of ML detection problem can be solved more efficiently by expanding the discrete feasible set into a continuous feasible region. The constraints in (16b) imply $\mathbf{z}^{T} \mathbf{z}=2 N$, which corresponds to a $2 N$-dimensional sphere centered at the origin with radius $\sqrt{2 N}$. Thus problem (16) can be converted to

$$
\begin{gathered}
\min \mathbf{z}^{T} \mathbf{Q z} \\
\text { subject to: } \mathbf{z}^{T} \mathbf{z}=2 N
\end{gathered}
$$

The receiver has knowledge that a particular QPSK symbol was transmitted on some pre-defined subcarriers, and this can be utilized to solve problem (17). Let $\mathbf{z}_{d}$ be the vector of the unknown transmitted data, $\Omega_{d}$ be the index set corresponding to $\mathbf{z}_{d}$, and $N_{d}$ be the size of $\mathbf{z}_{d}$. The corresponding pilot symbol vector is denoted as $\mathbf{z}_{p}, \Omega_{p}$ is the index set associated with $\mathbf{z}_{p}$, and $N_{p}$ is the size of $\mathbf{z}_{p}$. By substituting the known pilot tones $\mathbf{z}_{p}$ from the variable vector $\mathbf{z}, \mathbf{Q}_{d}$ and $\mathbf{q}_{d}$ can be obtained from $\mathbf{Q}$ with respect to $\Omega_{d}$ and $\Omega_{p}$, respectively. Consequently we have a quadratic optimization problem of the form

$$
\begin{aligned}
& \operatorname{minimize} \mathbf{z}_{d}^{T} \mathbf{Q}_{d} \mathbf{z}_{d}+\mathbf{q}_{d}^{T} \mathbf{z}_{d} \\
& \text { subject to: } \mathbf{z}_{d}^{T} \mathbf{z}_{d}=N_{d}
\end{aligned}
$$

\section{An Iterative Joint Semiblind Detection ALGORITHM}

\section{A. Basic algorithm}

The variables in (18) can be iteratively detected, where only some binary components of $\mathbf{z}_{d}$ are determined in each iteration by solving a corresponding non-combinatorial problem of type (18). Suppose that prior to the $i$ th iteration some binary components of vector $\mathbf{z}_{d}$ have been determined. Let $\mathbf{z}_{i}$ be the reduced-size vector that collects all undecided components of $\mathbf{z}_{d}, \Omega_{i}$ be the index set corresponding to $\mathbf{z}_{i}$, and $N_{i}$ be the size of $\mathbf{z}_{i}$ during the $i$ th iteration, respectively. By substituting the known binary components of $\mathbf{z}_{d}$ into (18), a reduced-size problem similar to (18) is obtained as

$$
\begin{gathered}
\operatorname{minimize} \mathbf{z}_{i}^{T} \mathbf{Q}_{i} \mathbf{z}_{i}+\mathbf{q}_{i}^{T} \mathbf{z}_{i} \\
\text { subject to: } \mathbf{z}_{i}^{T} \mathbf{z}_{i}=N_{i}
\end{gathered}
$$

The computational complexity is reduced by realizing that the variable set in problem (19) can be expressed with respect to a 2-dimensional subspace spanned by its steepest-descent direction (i.e., negative gradient of the objective function) and Newton direction. In doing so, we set

$$
\mathbf{z}_{i}=\eta_{1}^{(i)} \mathbf{v}_{1}^{(i)}+\eta_{2}^{(i)} \mathbf{v}_{2}^{(i)}
$$

where $\mathbf{v}_{1}^{(i)}=\mathbf{q}_{i}, \mathbf{v}_{2}^{(i)}=\mathbf{Q}_{i}^{-1} \mathbf{q}_{i}$, and $\eta_{1}^{(i)}, \eta_{2}^{(i)}$ are two scalar variables. Then (19) is converted to a 2-dimensional problem

$$
\begin{array}{r}
\operatorname{minimize} \boldsymbol{\eta}_{i}^{T} \boldsymbol{S}_{i} \boldsymbol{\eta}_{i}+\mathbf{p}_{i}^{T} \boldsymbol{\eta}_{i} \\
\text { subject to : } \boldsymbol{\eta}_{i}^{T} \mathbf{R}_{i} \boldsymbol{\eta}_{i}=N_{i}
\end{array}
$$

where $\boldsymbol{\eta}_{i}=\left[\eta_{1}^{(i)} \eta_{2}^{(i)}\right]^{T}, \mathbf{S}_{i}=\mathbf{V}_{i}^{T} \mathbf{Q}_{i} \mathbf{V}_{i}, \mathbf{p}_{i}=\mathbf{V}_{i}^{T} \mathbf{q}_{i}, \mathbf{R}_{i}=$ $\mathbf{V}_{i}^{T} \mathbf{V}_{i}$, and $\mathbf{V}_{i}=\left[\mathbf{v}_{1}^{(i)} \mathbf{v}_{2}^{(i)}\right]$. Consequently, the solution of (21) must satisfy the Karush-Kuhn-Tucker (KKT) conditions

$$
\begin{array}{r}
2 \mathbf{S}_{i} \boldsymbol{\eta}_{i}+\mathbf{p}_{i}+2 \lambda_{i} \mathbf{R}_{i} \boldsymbol{\eta}_{i}=0 \\
\boldsymbol{\eta}_{i}^{T} \mathbf{R}_{i} \boldsymbol{\eta}_{i}=N_{i}
\end{array}
$$

where $\lambda_{i}$ is a Lagrange multiplier at the $i$ th iteration. It follows that the optimal $\boldsymbol{\eta}_{i}$ can be obtained from (22a) as

$$
\boldsymbol{\eta}_{i}^{*}=-\frac{1}{2}\left(\mathbf{S}_{i}+\lambda_{i}^{*} \mathbf{R}_{i}\right)^{-1} \mathbf{p}_{i}
$$

Thus, (22b) can be rewritten as

$$
g\left(\lambda_{i}\right)=\sum_{k=0}^{N_{i}-1} \frac{\hat{p}_{k}^{2}}{\left(\lambda_{i}+s_{k}\right)^{2}}=4 N_{i}
$$

where $s_{k}$ is the $k$ th eigenvalue of $\hat{\mathbf{S}}_{i}=\mathbf{R}_{i}^{-\frac{1}{2}} \mathbf{S}_{i} \mathbf{R}_{i}^{-\frac{1}{2}}$, which admits an eigen-decomposition $\hat{\mathbf{S}}_{i}=\mathbf{U}_{i} \boldsymbol{\Sigma}_{i} \mathbf{U}_{i}^{T}$, and $\hat{p}_{k}$ is the $k$ th component of vector $\hat{\mathbf{p}}_{i}=\mathbf{U}_{i}^{T} \mathbf{R}_{i}^{-\frac{1}{2}} \mathbf{p}_{i}$. 
The only unknown variable $\lambda_{i}$ can be determined by solving the one-variable optimization problem

$$
\begin{aligned}
& \operatorname{minimize}\left|\sum_{k=0}^{N_{i}-1} \frac{\hat{p}_{k}^{2}}{\left(\lambda_{i}+s_{k}\right)^{2}}-4 N_{i}\right| \\
& \text { subject to: }-s_{l} \leq \lambda_{i} \leq \frac{\left\|\hat{\mathbf{p}}_{i}\right\|}{1.5 \sqrt{4 N_{i}}}-s_{l}
\end{aligned}
$$

with $s_{l}$ being the smallest value of $s_{k}$ such that $\hat{p}_{l} \neq 0$. The unique solution $\lambda_{i}^{*}$ of (25) can be effectively identified via bisection search.

Next, the magnitudes of the components of $\mathbf{z}_{i}^{*}$ are examined. If $\left|z_{k}^{*}\right|$ exceeds a given threshold $\rho=\alpha\left|z_{i}^{*}\right|_{\max }$, the corresponding variable is claimed as $\operatorname{sign}\left(z_{k}^{*}\right)$, otherwise component $z_{k}^{*}$ remains undetermined and will be considered as a design variable in the next iteration. Based on the components just detected, a QP problem similar to (18) with reduced size is produced where the vector $\mathbf{z}_{i}$ contains only the undetermined variables. This iterative process continues until all the variables have been identified to produce an estimate of the transmitted data.

Note that the evaluation of $\mathbf{Q}_{i}^{-1}$ is numerically intensive when its size is large. This can be alleviated using the wellknown formula for inverting a four-block matrix [15]. Thus the major portion of the computational complexity for the proposed algorithm is on the order of $O\left(k N^{2}\right)$ for each iteration, where $k$ denotes the average number of variables detected in one iteration and $k \ll N$ for a typical threshold value.

\section{B. Performance Enhancement by Low-Bit Descent Search}

In LBDS, a given binary sequence is associated with an objective function to be minimized. The search process evaluates, compares, and determines the optimal sign switches of a relatively small number of sequence components to yield maximum reduction in the objective function in (18). LBDS has been applied recently to various problems [16]. As will be demonstrated by simulation, the performance of the proposed algorithm can be considerably enhanced using 1-bit or 2-bit, or a combined 1-bit-and-2-bit LBDS, at an insignificant extra cost in computational complexity.

The iteration of LBDS is described as follows [16]. At each iteration, one-bit descent search evaluates $\mathbf{z}_{d} \odot \boldsymbol{\xi}$ (here $\odot$ denotes component-wise multiplication), where $\mathbf{z}_{d}$ is the vector defined in Section III, $\boldsymbol{\xi}=\tilde{\mathbf{Q}} \mathbf{z}_{d}+\mathbf{q}_{d} / 2$, and $\tilde{\mathbf{Q}}$ is generated from matrix $\mathbf{Q}_{d}$ with its diagonal components set to zero. Index $k^{*}$ is then identified as where the corresponding component $\xi_{k^{*}}$ has maximum value, and the sign of $z_{k^{*}}$ is switched to obtain an improved solution. Similarly, a 2-bit LBDS is performed by computing matrix $\mathbf{G}=\boldsymbol{\xi} e^{T}+\mathbf{e}^{T} \boldsymbol{\xi}-2 \mathbf{Q}_{d} \odot\left(\mathbf{z}_{d} \mathbf{z}_{d}^{T}\right)$, where e is the all-one vector. The index $\left(k^{*}, m^{*}\right)$ is identified as where the component $G_{k^{*}, m^{*}}$ reaches its maximum value, and an improved solution is then obtained by switching the signs of the $k^{*}$ th and $m^{*}$ th components of $\mathbf{z}_{d}^{*}$. The iterations continue until there is no further reduction in the objective function (18), at which point an estimate of the transmitted data is obtained.

\section{Simulation Results}

The proposed IJSD algorithm was applied to an OFDM system with $N=64$ subcarriers and a cyclic prefix of length $N_{c p}=N / 8$. The carrier frequency of the OFDM system was $5 \mathrm{GHz}$ and the bandwidth of the system was set to $3 \mathrm{MHz}$. The 6-ray COST $207 \mathrm{TU}$ model with power profile $[0.189,0.379,0.239,0.095,0.061,0.037]$ and delay profile $[0.0,0.2,0.5,1.6,2.3,5.0] \mu s$ was employed for the simulation. Each path is an independent complex Gaussian random process with Jake's Doppler spectrum. One or more pilot tones was utilized to compensate for the phase ambiguity, and BPSK or QPSK modulation was used for both the data and pilot symbols. The channel correlation matrix was assumed perfectly known at the receiver. The normalized Doppler frequency of the channel denoted as $f_{d} T_{s}$. The performance of the proposed joint detection algorithm was evaluated based on bit error rate (BER) and computational complexity, in comparison with a sphere decoder. The performance of the recursive detector [6] was used as the benchmark with the assumption of perfect channel state information (CSI).

The BER performance was evaluated with $f_{d} T_{s}=0.01$ for both BPSK and QPSK OFDM systems, as shown in Figs. 1 and 2. It can be observed that the IJSD algorithm provides comparable results to those of the sphere decoder with much less computational complexity. For example, for the QPSK OFDM system, at an $E_{b} / N_{0}$ of $30 \mathrm{~dB}$, the sphere algorithm has a BER of $6.4 \times 10^{-4}$, while the proposed algorithm with $\alpha=0.8$ achieves a BER of $3 \times 10^{-3}$ (with only $55 \%$ of the CPU time of the sphere decoder). This is improved to a BER of $8.5 \times 10^{-4}$ with LBDS (and $57 \%$ of the CPU time of the sphere decoder).

A larger $\alpha$ can provide better performance at a cost of increased computational complexity, as shown in Fig. 3. The proposed algorithm exhibits a performance loss due to a smaller threshold, but this loss can be avoided by performing LBDS with a slightly increased computational complexity. For the QPSK OFDM system with Doppler spread $f_{d} T_{s}=0.01$, the algorithm with $\alpha=0.5$ only requires $45 \%$ of the CPU time of the sphere decoder at an $E_{b} / N_{0}$ of $30 \mathrm{~dB}$. The corresponding BER with $\alpha=0.5$ is $4.5 \times 10^{-3}$. This can be improved to $9 \times 10^{-4}$ by performing LBDS with $50 \%$ of the CPU time of the sphere decoder. Table I summarizes the computational complexity of the IJSD algorithm relative to that of the sphere decoder for a QPSK OFDM system at $E_{b} / N_{0}=30 \mathrm{~dB}$, where the CPU time of the sphere decoder is normalized to one.

Simulations were also carried out to determine the impact of normalized doppler spread $f_{d} T_{s}$ on the system performance. The BER performances of the proposed algorithm for $f_{d} T_{s}=$ $0.001,0.005$, and 0.01 are depicted in Fig. 4. It can be observed that at lower SNR, the proposed algorithm provides similar performance for all values of Doppler spread. However, 
for smaller Doppler spreads, better performance was achieved at higher SNRs, e.g., at $E_{b} / N_{0}=30 \mathrm{~dB}$, a BER of $8.5 \times 10^{-4}$ was obtained for $f_{d} T_{s}=0.01$ using the proposed algorithm with LBDS, while a BER of $2.5 \times 10^{-4}$ can be achieved for $f_{d} T_{s}=0.001$.

Simulations were also carried out to determine the impact of the number of pilot tones on performance. The BER of the proposed algorithm with LBDS for $f_{d} T_{s}=0.01$ and 1, 2 and 4 pilot tones is shown in Fig. 5. It can be observed that there is an error floor at high signal-to-noise ratios with one pilot tone. The performance improves as the number of the pilot tones increases, with the error floor significantly reduced. For example, with one pilot tone, the proposed algorithm with LBDS achieves a BER of 0.015 at a $E_{b} / N_{0}$ of $30 \mathrm{~dB}$, while with 2 pilot tones, a BER of $2.2 \times 10^{-3}$ is obtained for the same $E_{b} / N_{0}$. The performance can be further improved to a BER of $8.5 \times 10^{-4}$ by utilizing 4 pilot tones.

\section{CONCLUSIONS}

A low complexity joint semiblind detection algorithm for OFDM systems over time-varying channels has been proposed. The joint semiblind problem was relaxed to a continuous nonconvex QP problem. An iterative method was then utilized to deduce a sequence of reduced-size QP problems by taking advantage of the pilot tones. These were solved by limiting the search in the 2-dimensional subspace to reduce the computational complexity. Furthermore, a low-bit descent search was employed to improve the system performance. Results were given which demonstrated that the proposed algorithm provides similar performance with lower computational complexity compared to that of a sphere decoder.

\section{REFERENCES}

[1] ETSI, "Digital video broadcasting: framing structure, channel coding, and modulation for digital terrestrial television," European Telecommunication Standard, ETS 300-744, Aug. 1997.

[2] ETSI, "Radio broadcasting systems: digital audio broadcasting to mobile, portable and fixed receivers," European Telecommunication Standard, ETS 300-401, Feb. 1995.

[3] IEEE 802.11, IEEE Standard for Wireless LAN: Medium Access Control (MAC) and Physical Layer (PHY) specifications, Nov. 1997.

[4] IEEE 802.16, IEEE Standard for Local and Metropolitan Area Networks: Air Interface for Fixed Broadband Wireless Access Systems, June 2004.

[5] X. Cai and G. B. Giannakis, "Bounding performance and suppressing intercarrier interference in wireless mobile OFDM," IEEE Trans. Commun., vol. 51, no. 12, pp. 2047-2056, Dec. 2003.

[6] Y. H. Zhang, W.-S. Lu, and T. A. Gulliver, "A successive intercarrier interference reduction algorithm for OFDM systems," Proc. IEEE Int. Conf. Commun., June 2007.

[7] A. R. S. Bahai, B. R. Saltzberg, and M. Ergen, Multicarrier Digital Communications: Theory and Applications of OFDM, Springer, 2004.

[8] Y.-S. Choi, P. J. Voltz, and F. A. Cassara, "On channel estimation and detection for multicarrier signals in fast and selective Rayleigh fading channels," IEEE Trans. Commun., vol. 49, no. 8, pp. 1375-1387, Aug. 2001.

[9] H. Vikalo, B. Hassibi, and P. Stoica, "Efficient joint maximum-likelihood channel estimation and signal detection," IEEE Trans. Wireless Commun., vol. 5, no. 7, pp. 1838-1845, July 2006.
[10] M.-X. Chang and Y. T. Su, "Blind and semiblind detection of OFDM signals in fading channels," IEEE Trans. Commun., vol. 52, no. 5, pp. 744-754, May 2004.

[11] T. Cui and C. Tellambura, "Semi-blind equalization for OFDM systems over fast fading channels," Proc. IEEE Int. Conf. Commun., pp. 1137-1141, May 2005.

[12] J. G. Proakis, Digital Communications, 4th Ed., McGraw-Hill, 2001.

[13] W. C. Jakes, Microwave Mobile Communications, Wiley, 1974.

[14] E. Viterbo and J. Boutros, "A universal lattice code decoder for fading channels," IEEE Trans. Inform. Theory, vol. 45, no. 5, pp. 1639-1642, July 1999.

[15] R. A. Horn and C. R. Johnson, Matrix Analysis, Cambridge University Press, 1985.

[16] W.-S. Lu, "Design of FIR digital filters with discrete coefficients via convex programming," Proc. IEEE ISCAS, pp. 1831-1834, May 2005.

TABLE I

COMPUTATIONAL COMPLEXITY COMPARISON

\begin{tabular}{|c|c|c|}
\hline \multirow{2}{*}{ Algorithm } & \multicolumn{2}{|c|}{ Normalized CPU time } \\
\cline { 2 - 3 } & $\alpha=0.8$ & $\alpha=0.5$ \\
\hline IJSD Without LBDS & 0.55 & 0.45 \\
\hline IJSD With LBDS & 0.57 & 0.5 \\
\hline Sphere decoder & \multicolumn{2}{|c|}{1} \\
\hline
\end{tabular}

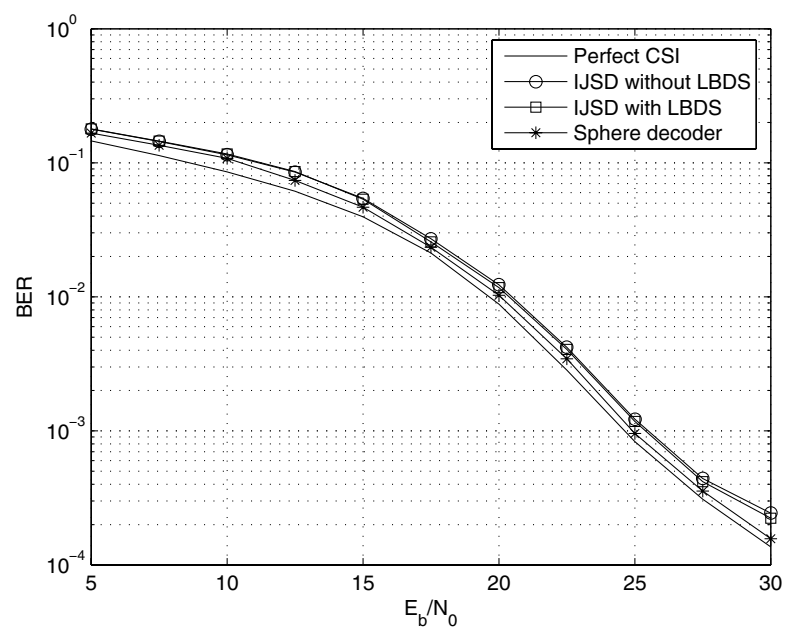

Fig. 1. BPSK OFDM system performance with $f_{d} T_{s}=0.01, \alpha=0.8$ and 4 pilot tones. 


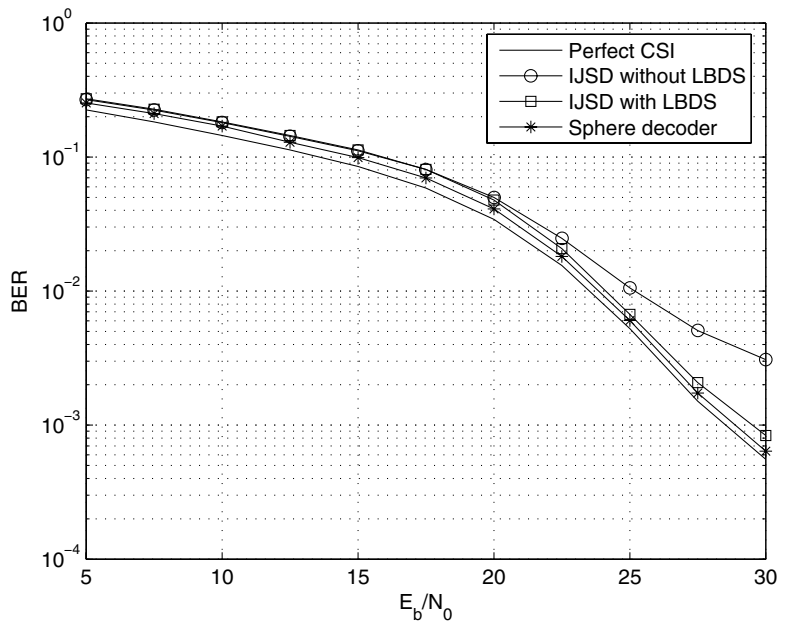

Fig. 2. QPSK OFDM system performance with $f_{d} T_{s}=0.01, \alpha=0.8$ and 4 pilot tones.

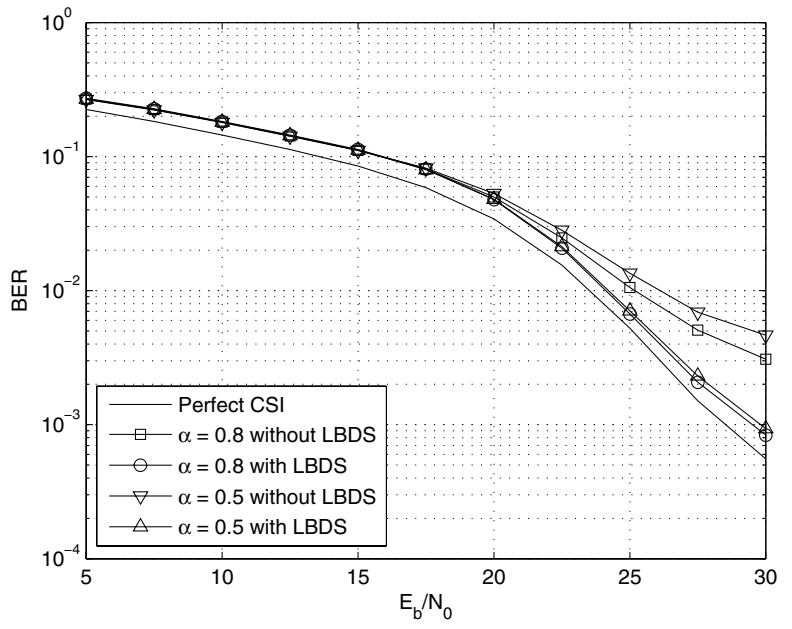

Fig. 3. QPSK OFDM system performance with $f_{d} T_{s}=0.01,4$ pilot tones and various thresholds using the proposed IJSD algorithm.

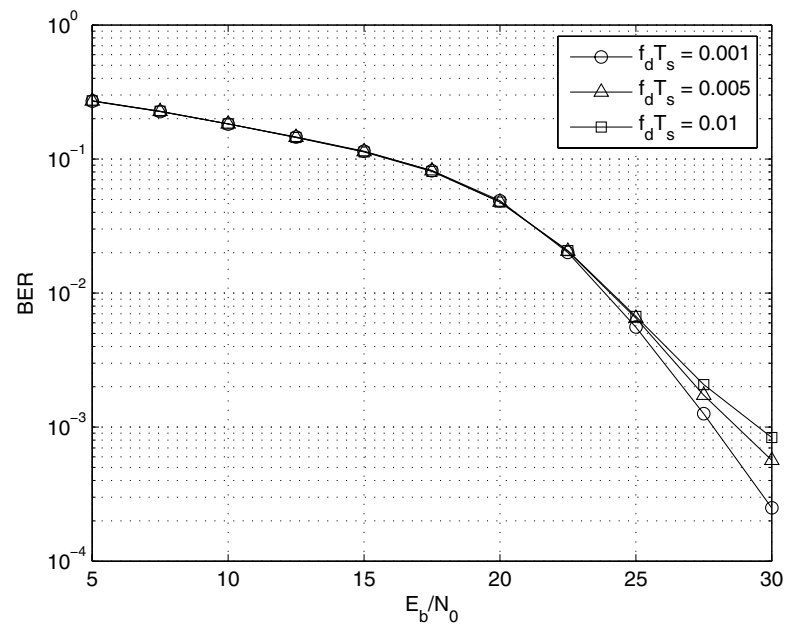

Fig. 4. QPSK OFDM system performance with $\alpha=0.8,4$ pilot tones and various Doppler spreads using the proposed IJSD algorithm with LBDS.

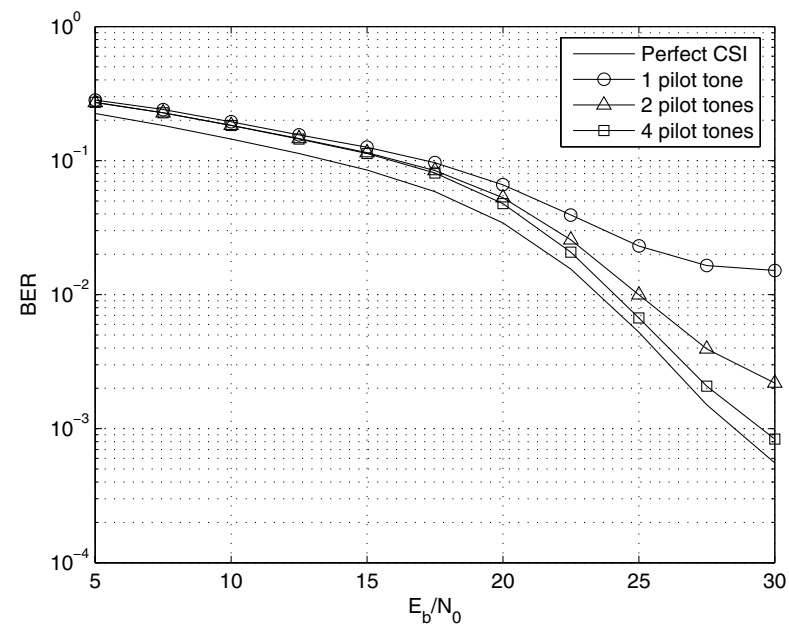

Fig. 5. QPSK OFDM system performance with $f_{d} T_{s}=0.01, \alpha=0.8$ and various pilot tones using the proposed IJSD algorithm with LBDS. 